\title{
Conocimiento popular de los mamíferos del Parque Nacional Barra Honda, Nicoya, Costa Rica
}

\section{Popular knowledge of the mammals of Barra Honda National Park, Nicoya, Costa Rica}

\section{Conhecimento popular dos mamíferos do Parque Nacional Barra Honda, Nicoya, Costa Rica}

Maikol Castillo-Chinchilla

maikol.castillo.chinchilla@una.cr

Escuela de Ciencias Biológicas,

Universidad Nacional,

Heredia, Costa Rica.

Orcid: https://orcid.org/0000-0002-6823-0326

Lilliana Piedra-Castro

lilliana.piedra.castro@una.cr

Escuela de Ciencias Biológicas,

Universidad Nacional,

Heredia, Costa Rica.

Orcid: https://orcid.org/0000-0003-4878-1531
Iván Sandoval-Hernández

ivan.sandoval.hernandez@una.cr

Escuela de Ciencias Biológicas,

Universidad Nacional,

Heredia, Costa Rica.

Orcid: https://orcid.org/0000-0002-0495-1829

\author{
José Pablo Carvajal-Sánchez \\ jose.carvajal.sanchez@una.cr \\ Instituto Internacional en Manejo y Conservación de \\ Vida Silvestre, \\ Universidad Nacional, \\ Heredia, Costa Rica. \\ Orcid: https://orcid.org/0000-0002-3173-1063
}

Recibido-Received: 18/abr/2017 • Corregido-Corrected: 17/ago/2017

Aceptado-Accepted: 1/dic/2017 • Publicado-Published: 31/jul/2018

\section{Resumen}

En el mundo, las actividades antrópicas y la pérdida de hábitat tienen efectos negativos sobre la flora y fauna. Se ha comprobado que el estudio del conocimiento popular de grupos faunísticos representa un recurso valioso para la toma de decisiones. El objetivo de este estudio fue evaluar el conocimiento popular sobre los mamíferos terrestres en seis poblados circundantes al Parque Nacional Barra Honda (PNBH), Nicoya, Costa Rica. El análisis de las encuestas se realizó tomando en cuenta la frecuencia y los porcentajes obtenidos en relación con el conocimiento popular, la diversidad de especies, las percepciones sobre los mamíferos terrestres y las actividades de cacería. Los entrevistados mencionaron que, en las áreas externas al PNBH, se pueden observar entre 14 y 18 mamíferos, donde se destaca al puma (Puma concolor), tolomuco (Eira barbara), tepezcuintle (Cuniculus paca) y al venado cola blanca 
(Odocoileus virginianus). Alrededor del $50 \%$ de los entrevistados identificaron el tepezcuintle y al venado cola blanca como especies que son cazadas con mayor frecuencia. Algunos de los mamíferos identificados por los pobladores de las áreas circundantes al PNBH son catalogados como perjudiciales para los cultivos, animales de granja y ganado, por lo que son cazados de manera indiscriminada.

Palabras claves: hábitat; mamíferos; especies; conocimiento popular

\section{Abstract}

Human activities and loss of habitat can have negative effects on flora and fauna. It has been shown that the study of popular knowledge of faunal groups represents a valuable resource for decision making. The objective of this study is to carry out an analysis of popular knowledge about terrestrial mammals in six villages surrounding Barra Honda National Park, Nicoya (BHNP), Costa Rica. The analysis of survey results was carried out taking into account the frequencies and percentages obtained in relation to popular knowledge, species diversity, perceptions on terrestrial mammals and hunting activities. Interviewees mentioned that in areas outside the BHNP, 14 to 18 species of mammals can be observed, including puma (Puma concolor), tolomuco (Eira barbara), tepezcuintle (Cuniculus paca) and white-tailed deer (Odocoileus virginianus). About $50 \%$ of respondents identified tepezcuintle and white-tailed deer as the species that are most frequently hunted. Some of the mammals identified by the villagers in the areas surrounding the BHNP are regarded as harmful to crops, farm animals and cattle, and are hunted indiscriminately.

Keywords: habitat; mammals; species; popular knowledge

\section{Resumo}

No mundo, as atividades antrópicas e a perda de hábitat têm efeitos negativos sobre a flora e fauna. Já foi comprovado que o estudo do conhecimento popular de grupos faunísticos representa um recurso valioso para a tomada de decisões. O objetivo deste estudo foi avaliar o conhecimento popular sobre os mamíferos terrestres em seis povoados circunvizinhos ao Parque Nacional Barra Honda (PNBH), Nicoya, Costa Rica. A análise das enquetes foi realizada levando em conta a frequência e as porcentagens obtidas com relação ao conhecimento popular, a diversidade de espécies, as percepções sobre os mamíferos terrestres e as atividades de caça. Os entrevistados mencionaram que nas áreas externas ao PNBH podem ser observados entre 14 e 18 mamíferos, dentre os quais se destacam a onça parda (Puma concolor), a papa-mel (Eira barbara), a paca (Cuniculus paca) e o veado-de-cauda-branca (Odocoileus virginianus). Aproximadamente $50 \%$ dos entrevistados identificaram a paca e o veado-de-cauda-branca como espécies que são caçadas com maior frequência. Alguns dos mamíferos identificados pelos moradores das áreas circundantes ao PNBH são catalogados como prejudiciais aos cultivos, animais de granja e gado, razão pela qual são caçados de maneira indiscriminada.

Palavras-chaves: hábitat; mamíferos; espécies; conhecimento popular 
$\mathrm{E}$ n el mundo, las actividades antrópicas como la cacería furtiva, la silvicultura intensiva, la deforestación y el urbanismo descontrolado se han señalado como factores que provocan la modificación de los hábitats naturales, que junto con aspectos económicos y sociales favorecen un escenario complejo que puede ocasionar la desaparición local de especies de flora y fauna (Troche y Guarachi, 2001; Lindenmayer et al., 2002; Rodas-Trejo, Ocampo-Gonzáles y Coutiño-Hernández, 2014).

De ahí que hoy existe la necesidad de obtener información mediante distintos métodos que favorezcan la toma de decisiones. Esta indagación debe estar orientada para la determinación del estado de salud de las poblaciones naturales y de las interacciones que estas mantienen con los asentamientos humanos, los cuales pueden ser factor clave en los cambios espaciales y temporales que sufran (Nupp y Swihart, 1996; Stevens, Polus, Wesselingh, Schtickzelle y Baguette, 2004; Schelhas, 2007).

Dehustua, (2008) y Galindo, (2012) consideran que el conocimiento popular sobre grupos faunísticos representa un recurso valioso para el levantamiento de información, dado que se relaciona con experiencias pasadas y con cuantiosas horas de observación del comportamiento de la fauna en su ambiente natural. Por ello, recurrir a la recolección de este tipo de información en las zonas circundantes al PNBH representa un insumo fundamental que complementa las investigaciones de grupos como los mamíferos.

De igual manera, el conocimiento popular representa uno de los elementos de importancia en los estudios mastozoológicos en el nivel mundial, puesto que permiten obtener información sobre aspectos ecológicos, biológicos y económicos que pueden influir en las estrategias de conservación y manejo integral de los recursos naturales (Rodas-Trejo et al., 2014).

En el caso de Costa Rica, la investigación respecto a los mamíferos que habitan en algunas de sus áreas protegidas es escasa, por lo que estudios en esta temática permiten analizar la situación actual de la fauna y contribuyen con las acciones de los tomadores de decisión para asegurar la protección de la mastofauna. Este es el caso del Parque Nacional Barra Honda (PNBH), donde se requieren datos que contribuyan a evaluar las poblaciones de los mamíferos y de las posibles interacciones de estos con los asentamientos humanos circundantes.

Motivados por esta ausencia de información en el PNBH, el objetivo del presente estudio fue evaluar el conocimiento de las comunidades circundantes a dicho parque, ubicado en Nicoya, Costa Rica, respecto a los mamíferos terrestres, como contribución a la gestión del área protegida.

\section{Metodología}

El estudio se realizó en las localidades circundantes con el límite externo del Parque Nacional Barra Honda, ubicado en la península de Nicoya a $22 \mathrm{~km}$ noroeste del cantón de Nicoya, Guanacaste, Costa Rica (10¹0’84 N; 085²1'52 O). Esta área protegida tiene una extensión territorial de 2297 hectáreas y resguarda bosque tropical seco con transición a bosque húmedo, caracterizado por una época lluviosa que va de mayo a noviembre y una seca entre diciembre y abril. Se encuentra rodeada de los poblados Quebrada Honda (QH), Caballito (CB), Corralillo (CR), El Flor (EF), Santa Ana (SA) y Barra Honda (BH), entre los meses de julio y noviembre del 2011, cuando se realizaron encuestas semiestructuradas (ver Figura 1). 

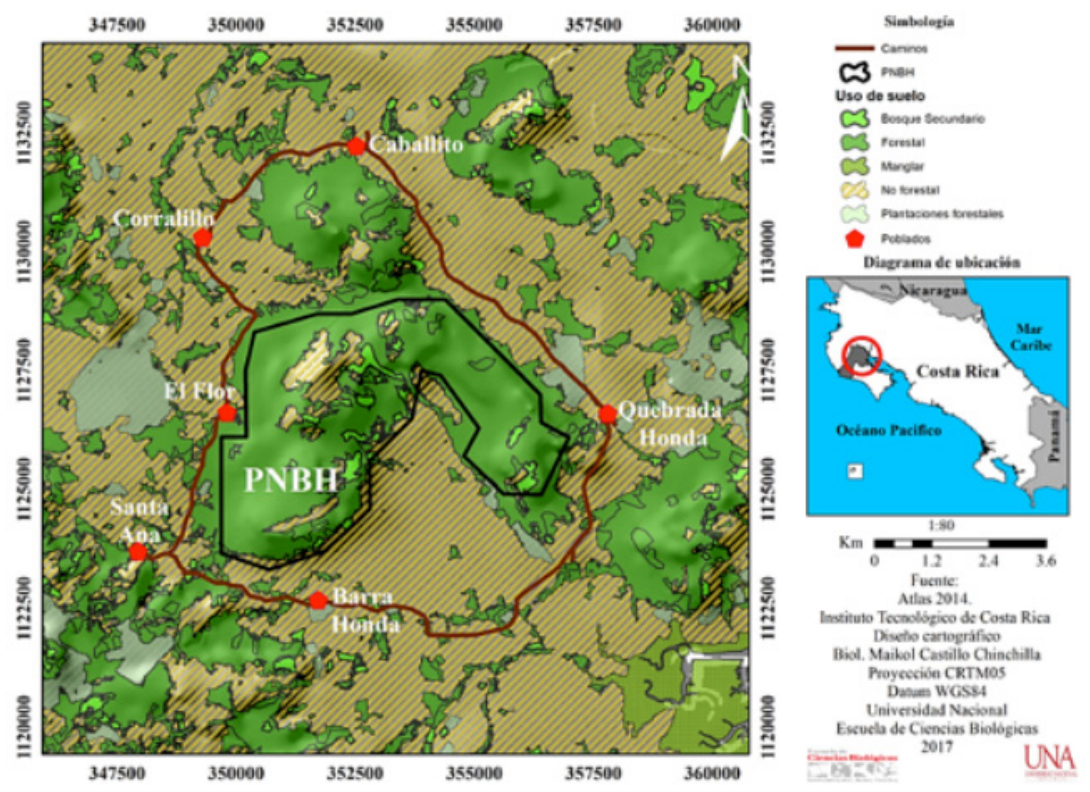

Figura 1. Ubicación de las comunidades limitrofes con el Parque Nacional Barra Honda, Nicoya, Costa Rica. Fuente propia de la investigación.

Los criterios que se utilizaron para seleccionar estas comunidades incluyeron la cercanía al área protegida, el tamaño, la accesibilidad por los caminos y las rutas de autobús, así como un estudio previo realizado por Pacheco (2003). Los grupos meta correspondieron a jefas de hogar, comerciantes, agricultores, mayores a los 15 años.

En cinco visitas de campo se aplicaron 36 encuestas semiestructuradas que correspondieron al $45 \%$ de la muestra total esperada, las cuales se llevaron a cabo de manera aleatoria en las unidades habitacionales en donde se ubicó sujetos muestreales que evidenciaran anuencia a colaborar con la investigación. La estimación de la muestra se basó en la referencia de la cantidad de habitaciones que se contabilizaron en imágenes aéreas de Google Earth V6 2011.

Dicho instrumento se subdividió por temáticas relacionadas con: a) el conocimiento popular sobre los mamíferos de la zona, b) diversidad de especies, c) las percepciones sobre los mamíferos, d) activida- des de cacería en el PNBH. Como apoyo a la identificación de mamíferos durante las encuestas, se utilizaron las guías de campo de Reid (1997), Carrillo, Wong y Sáenz (2002), Aranda (2000) y Wainwright (2005), con las cuales los encuestados identificaron características que permiten la determinación de cada especie observada (Anexo 1).

Los datos se analizaron con el paquete estadístico de Excel 2013, para obtener los porcentajes por respuesta, en los diversos componentes o áreas que se evaluaron (Sampieri, Collado y Lucio, 2010).

\section{Análisis y resultados}

En las seis localidades circundantes al PNBH, se mencionó que se pueden encontrar entre 14 y 19 especies de mamíferos que corresponden a alrededor del $92 \%$ de los mamíferos terrestres reportados en el parque (ver Tabla 1). Entre las especies que mencionaron los encuestados, se destacó el tepezcuintle (Cuniculus paca), el 
puma (Puma concolor), el tolomuco (Eira barbara) y el saíno (Pecari tajacu), como las especies más difíciles de observar en los alrededores del parque (ver Gráfica 1).

Tabla 1

Lista de especies reportadas en los poblados de Quebrada Honda (QH), Caballito (CB), Corralillo (CR), El Flor (EF), Santa Ana (SA) y Barra Honda (BH)

\begin{tabular}{|c|c|c|c|c|}
\hline \multirow[t]{2}{*}{ Localidad } & \multirow[t]{2}{*}{ Especie } & \multirow[t]{2}{*}{ Nombre común } & \multicolumn{2}{|c|}{\begin{tabular}{|l}
$\begin{array}{l}\text { Estado de con- } \\
\text { servación }\end{array}$ \\
\end{tabular}} \\
\hline & & & UICN & CITES \\
\hline $\mathrm{QH}, \mathrm{CB}, \mathrm{CR}, \mathrm{SA}, \mathrm{BH}, \mathrm{EF}$ & Canis latrans & Coyote & $\mathbf{P M}$ & $*$ \\
\hline $\mathrm{QH}, \mathrm{CB}, \mathrm{CR}, \mathrm{BH}, \mathrm{EF}$ & Cuniculus paca & Tepezcuintle & $\mathbf{P M}$ & $*$ \\
\hline $\mathrm{QH}, \mathrm{CB}, \mathrm{CR}, \mathrm{SA}, \mathrm{BH}$ & Dasyprocta punctata & Cherenga & PM & * \\
\hline $\mathrm{QH}, \mathrm{CR}, \mathrm{SA}, \mathrm{BH}, \mathrm{EF}$ & Dasypus novemcinctus & Armadillo & PM & * \\
\hline $\mathrm{CB}, \mathrm{SA}, \mathrm{BH}, \mathrm{EF}$ & Eira barbara & Tolomuco & PM & * \\
\hline $\mathrm{CB}, \mathrm{CR}, \mathrm{SA}, \mathrm{EF}$ & Conepatus semistriatus & Zorro hediondo & PM & * \\
\hline $\mathrm{CB}, \mathrm{SA}, \mathrm{EF}$ & Leopardus pardalis & Manigordo & PM & I \\
\hline $\mathrm{QH}, \mathrm{CB}, \mathrm{CR}, \mathrm{BH}, \mathrm{EF}$ & Nasua narica & Pizote & PM & $*$ \\
\hline $\mathrm{QH}, \mathrm{CR}, \mathrm{SA}, \mathrm{BH}$ & Odocoileus virginianus & $\begin{array}{|ll|}\begin{array}{l}\text { Venado } \\
\text { blanca }\end{array} & \text { cola } \\
\end{array}$ & $\mathbf{P M}$ & $*$ \\
\hline $\mathrm{QH}, \mathrm{CB}, \mathrm{CR}, \mathrm{EF}$ & Procyon lottor & Mapache & PM & $*$ \\
\hline $\mathrm{CB}, \mathrm{BH}, \mathrm{EF}$ & Puma concolor & León de montaña & PM & I \\
\hline $\mathrm{QH}, \mathrm{CB}, \mathrm{CR}, \mathrm{SA}, \mathrm{BH}$ & Sylvilagus floridanus & Conejo & PM & $*$ \\
\hline $\mathrm{CB}, \mathrm{CR}, \mathrm{SA}, \mathrm{EF}$ & Tamandua mexicana & Oso de chaleco & PM & * \\
\hline $\mathrm{CB}, \mathrm{CR}, \mathrm{SA}, \mathrm{BH}, \mathrm{EF}$ & Pecari tajacu & Saíno & PM & II \\
\hline $\mathrm{QH}, \mathrm{CB}, \mathrm{SA}, \mathrm{BH}, \mathrm{EF}$ & Alouatta palliata & Mono congo & PM & I \\
\hline $\mathrm{QH}, \mathrm{CR}, \mathrm{SA}, \mathrm{BH}, \mathrm{EF}$ & Cebus imitator & Mono carablanca & $\mathbf{P M}$ & II \\
\hline $\mathrm{QH}, \mathrm{CB}, \mathrm{CR}, \mathrm{SA}, \mathrm{BH}$ & Didelphis sp. & Zorro pelón & PM & * \\
\hline $\mathrm{QH}, \mathrm{CB}, \mathrm{CR}, \mathrm{SA}, \mathrm{BH}$ & Sciurus variegatoides & Ardilla & PM & $*$ \\
\hline $\mathrm{CB}, \mathrm{SA}, \mathrm{CR}, \mathrm{EF}$ & Sphiggurus mexicanus & Puerco espín & PM & * \\
\hline
\end{tabular}




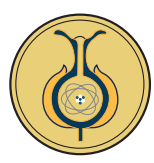

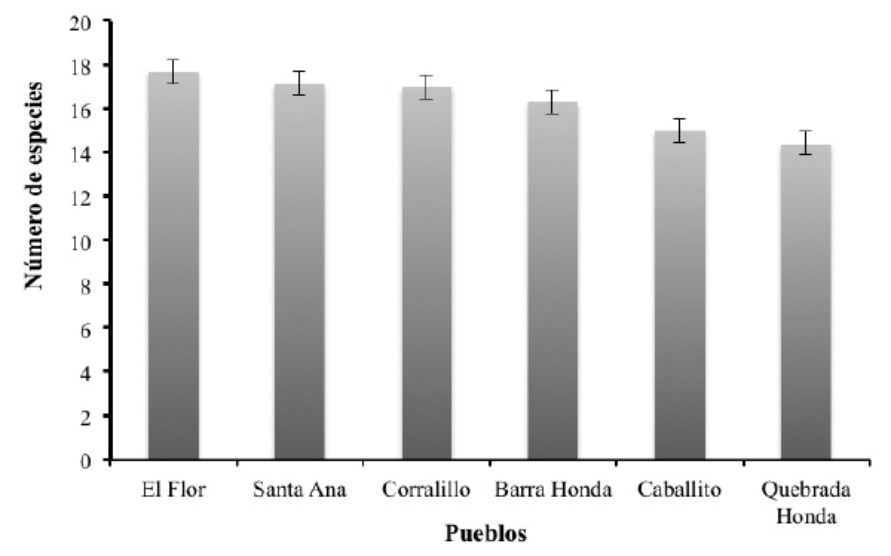

Gráfica 1

Número de especies identificadas en pueblos circundantes al Parque Nacional Barra Honda, Nicoya, Costa Rica Nota: Fuente propia de la investigación.

Los pobladores consideran que al menos el $61 \%$ de los mamíferos que existen en las áreas circundantes al PNBH son perjudiciales, principalmente por los problemas que ocasionan en cultivos, por la depredación de animales de granja y ganado. Las especies que fueron mencionadas por los participantes, con mayor frecuencia en estas categorías, fueron el mapache ( $P$. lottor $)$, el pizote ( $N$. narica), el coyote (C. latrans), el venado (O. virginianus) y los zorros (Didelphis sp.) (ver Gráfica 2).

Con respecto a la cacería, se pudo identificar que las razones que motivan esta práctica en zonas que bordean el $\mathrm{PNBH}$ son principalmente el deporte (39\%), la subsistencia $(28 \%) \mathrm{y}$, en pocos casos, el comercio $(3 \%)$, siendo el venado cola blanca $(O$. virginianus) y el tepezcuintle (C. paca) las especies capturadas con mayor frecuencia (ver Gráfica 3).

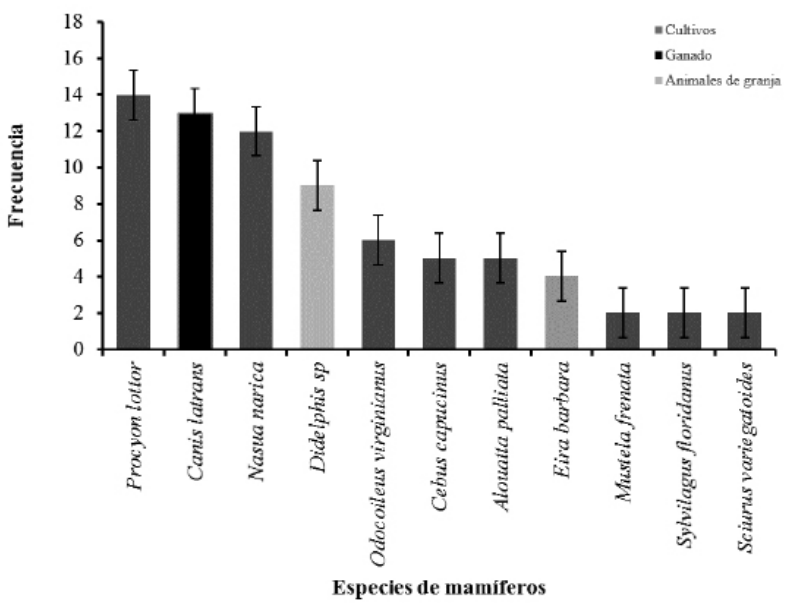

Gráfica 2

Mamíferos considerados perjudiciales

en las áreas circundantes del Parque

Nacional Barra Honda, Nicoya, Costa

Rica

Nota: Fuente propia de la investigación.

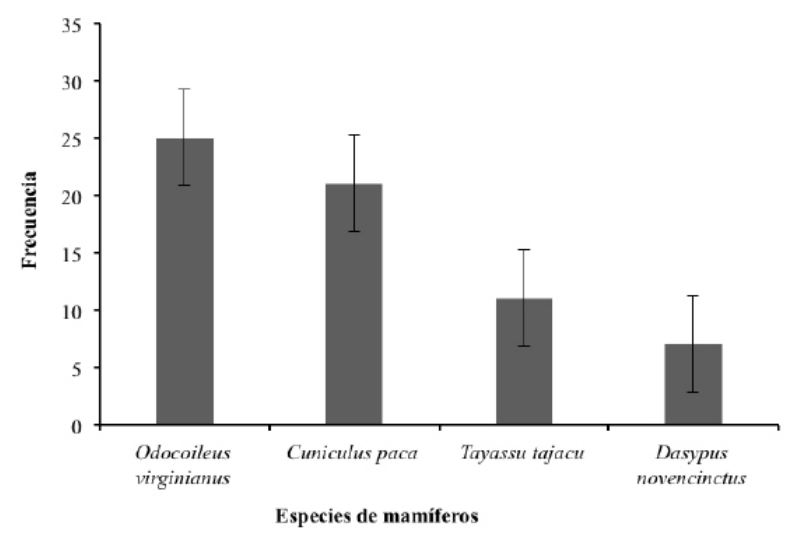

Gráfica 3

Mamíferos con valor cinegético para los locales del Parque Nacional Barra Honda, Nicoya, Costa Rica

Nota: Fuente propia de la investigación. 
Por otro lado, los encuestados perciben como los mamíferos más abundantes de la zona a especies gregarias como el mono congo (A. palliata) y a otras solitarias como el armadillo ( $D$. novencimctus), el venado cola blanca (O. virginianus) y las ardillas (Sciurus variegatoides) (ver Gráfica 4).

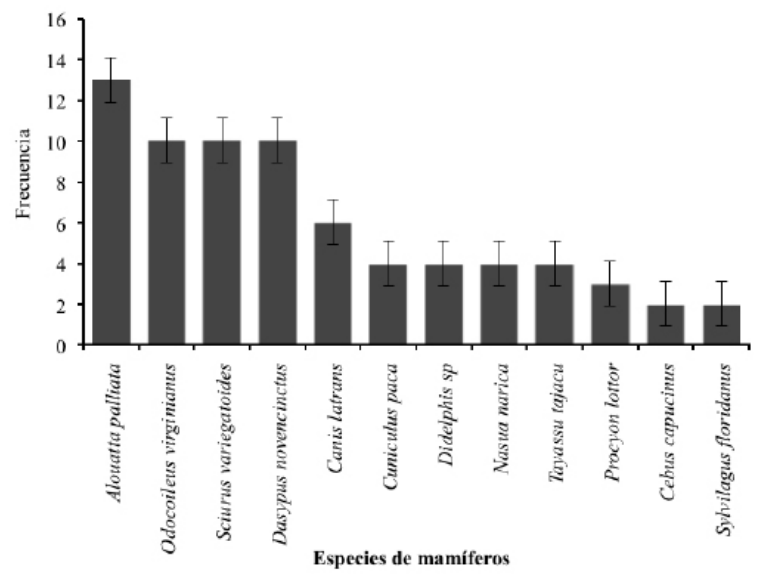

Gráfica 4

Percepción de la abundancia de los mamíferos del Parque Nacional Barra Honda, Nicoya, Costa Rica

Nota: Fuente propia de la investigación.

\section{Discusión}

El uso de las guías de campo en las encuestas permitió que los pobladores de las áreas circundantes al PNBH brindaran información más precisa sobre presencia de mamíferos, lo cual es sustentado por Flores-Manzanero, González-Pérez, Vásquez-Dávila y Manzanero-Medina (2013), quienes señalan que los habitantes de una región determinada mejoran la descripción de especies al emplear como apoyo medios visuales, dibujos o fotografías.

En ese sentido, el número de especies que identificaron los pobladores es coincidente con un estudio previo y registros de avistamientos que mantienen los funcio- narios del parque (Pacheco, 2003). Un hallazgo importante es que se mencionara que en la zona existen felinos grandes, como el puma ( $P$. concolor), el cual podría estar utilizando el área protegida como sitio de paso hacia algunas zonas boscosas de la provincia, o bien como sitio de alimentación, dada la presencia de algunas de sus presas comunes en la zona, como el saíno (P. tajacu) o el venado cola blanca (O. virginianus) (Reid, 1997; Carrillo et al., 2002; Bustamante, Moreno, Rossel y Artavia, 2014).

Con respecto a la baja diversidad de mamíferos, los entrevistados señalaron que la modificación y pérdida del hábitat que ocurrió en las áreas cercanas al PNBH ocasionó reducciones importantes de la cobertura boscosa, como consecuencia de la expansión de la frontera agrícola y actividades ganaderas, establecidas en sitios que deberían funcionar principalmente como zona de amortiguamiento (Pacheco, 2003).

Según autores como Escamilla, San Vicente, Sosa y Galindo-Leal (2000); Salóm-Pérez, Carrillo, Sáenz y Mora (2007); Ávila-Nájera, Rosas-Rosas, Tarango-Arámbula, Martínez-Montoya y Santoyo-Brito (2011), y García (2011), las modificaciones del entorno de un área protegida pueden incrementar el riesgo de extinción para la mastofauna, hecho que afectaría principalmente a especies presentes en el PNBH, que tienen alto grado de especialización, poca movilidad o gran tamaño como los felinos, los cuales, además, son susceptibles a alteraciones del hábitat y requieren de cierto estándar de calidad en las áreas que podrían utilizar para desplazarse.

Aunado a lo anterior, la falta de conectividad con otras áreas protegidas y la carencia de extensiones de bosque adecuadas podrían ser parte de las acciones por las que en el PNBH y sus alrededores parecen ha- 
ber desaparecido mamíferos grandes como el jaguar $(P$. onca) o jaguaroundi ( $P$. yaguarondi), puesto que no reúnen las condiciones mínimas para su supervivencia. Esto último es parte de las razones por las que los encuestados no las mencionaron en la lista de especies presentes (Beier, 1993; Amit, 2006; Salóm-Pérez et al., 2007; Bustamante, 2008, Ávila-Nájera et al., 2011).

Desde este punto de vista, las alteraciones presentadas en el entorno del área protegida podrían haber afectado directamente a la mastofauna presente, al disminuir las opciones de desplazamiento para las especies que requieren trasladarse hacia otros lugares en busca de recursos, que disminuyen durante el ciclo anual por efectos naturales (Nupp y Swihart, 1996; Stevens et al., 2004; Schelhas, 2007).

En el PNBH, parece existir una elevada dependencia de los mamíferos por los terrenos cercanos al área protegida, en los cuales intentan satisfacer necesidades de espacio, alimentación y refugio, llevando, de esta forma, a conflictos con los pobladores. Lo anterior es concordante con lo mencionado por Parra-Colorado, Botero-Botero y Saavedra-Rodríguez (2014), quienes indican que el reconocimiento y la interacción de la fauna con el hombre están relacionados con el inadecuado uso de la tierra, como el que ocurrió en los linderos del parque.

Por otro lado, interacciones humanas, como la cacería y el tamaño del parque, pueden tener efectos sobre la composición de mamíferos, ya sea por la presión que se genera sobre algunas especies en particular, o bien, por la disminución de los recursos del hábitat. A esto debe sumarse que en Costa Rica hace falta investigación sobre la ecología del paisaje, el estado de las poblaciones, evaluaciones de la efectividad de la conectividad entre áreas protegidas, que permita tomar medidas de gestión y manejo para las especies (Sandoval y Castillo, 2011).

En este sentido y tal como lo menciona Parra-Colorado et al. (2014), las interacciones de humano-mamíferos, especialmente con los carnívoros, detonan en conflictos generados por el perjuicio que ocasionan estos a los animales domésticos o a los cultivos. En el caso de la presente investigación, se evidencia que los mamíferos detectados son cazados por deporte; son blanco de este grupo todos aquellos que ocasionan daños o son considerados perjudiciales para los intereses humanos (Echegaray, 2004).

En relación con la cacería, Tlapaya y Gallina (2010) indican que este tipo de actividades son frecuentes en las comunidades rurales, cercanas a las áreas protegidas, en donde los cazadores obtienen presas con mayor facilidad, debido a que, por ser sitios en los que se realizan acciones de protección de la fauna silvestre, se facilita la captura de esta cuando el recurso humano es insuficiente para cuidar las áreas de protección.

La cacería, además de ser una actividad ilegal en Costa Rica, puede alterar el equilibrio ecológico, desestabilizar las cadenas alimenticias, y provocar que mamíferos frecuenten los pueblos cercanos en busca de las presas que el parque no provee. Se ha documentado que al presentarse prácticas como estas se presenta una menor disponibilidad de presas silvestres, lo cual conlleva un incremento en el consumo de animales domésticos por carnívoros como los felinos, coyotes, pizotes, mapaches (Gallardo, Nuñez, Pacheco y Ruiz-García, 2009; Ávila-Nájera et al., 2011, Parra-Colorado et al., 2014).

Esto tiende a convertirse en un efecto redundante, se caza sin conocer el máximo rendimiento sostenible, se pone en riesgo la sobrevivencia en el largo plazo, por lo que 
se convierte en uno de los factores que presiona la vida silvestre y puede ser considerado un indicador de la efectividad que tienen las acciones de manejo y conservación en las áreas protegidas.

También debe considerarse que los patrones de cacería presentados en los asentamientos humanos que bordean el PNBH son similares a los que se dan en bosques tropicales, en donde los mamíferos ocupan el primer lugar entre los animales cazados, lo cual parece estar relacionado con el mayor aporte de biomasa para consumo personal, o bien con fines comerciales (Racero-Casarrubia, Vidal, Ruiz y Ballesteros, 2008; Fa, Aguilar, Moya, Duarte y Yañez, 2013).

Por estas razones, se considera que el conocimiento de los pobladores de las áreas circundantes al PNBH es valioso por el aporte que genera. Además, este tipo de investigaciones que abordan temas relacionados con el saber popular deben mantener prioridad en la agenda ambiental actual, ya que son indispensables en el desarrollo de la valoración sobre el uso de la biodiversidad (Gil y Guiascón, 2015).

Finalmente, los pobladores consideran como abundantes a especies como el congo o el venado cola blanca, que se observan con relativa facilidad en la parte interna y externa del área protegida. Dicha percepción de la abundancia podría estar relacionada con el reducido tamaño que tiene el parque y la considerable movilidad de las especies mencionadas. Se trata de mamíferos de tamaño mediano y de comportamiento poco esquivo, tolerantes a la presencia humana, lo cual facilita los encuentros con personas y esto podría incidir en la percepción de la abundancia (Gallardo et al., 2009).

Debido a lo anterior, se debe manejar con precaución este tipo de datos de abundancia, que aportan los pobladores, hasta que se puedan realizar investigaciones con diferentes técnicas de muestreo que permitan determinar la condición actual de las poblaciones de mastofauna que mantiene el PNBH.

Tal como indican los resultados de la investigación, se encontraron diferencias y algunas similitudes entre la percepción que mantienen los pobladores de áreas circundantes al PNBH sobre los grupos faunísticos, lo cual puede relacionarse con sus experiencias pasadas e historia de vida en la zona, aspectos que aportan información valiosa a los estudios de mamíferos terrestres.

\section{Conclusiones}

En el PNBH existen especies evasivas difíciles de observar en el área protegida, sin embargo, algunas de ellas fueron identificadas por los pobladores de las áreas circundantes.

Los pobladores perciben a algunos de los mamíferos como perjudiciales para los cultivos, animales de granja y ganado, por lo que son cazados de manera indiscriminada.

Las comunidades cercanas al PNBH requieren programas de educación ambiental que contribuyan en la protección de los recursos y que favorezcan los procesos de involucramiento de pobladores con el manejo del AP.

\section{Agradecimientos}

A todo el personal del Parque Nacional Barra Honda, por la totalidad del apoyo brindado durante el desarrollo de la investigación, y a todas aquellas personas que colaboraron activamente en la mejora del documento. 


\section{Referencias}

Amit, R. (2006). El jaguar (Panthera onca) en el sector San Cristóbal del Área de Conservación Guanacaste-Costa Rica: densidad, abundancia de presas y depredación de ganado. (Tesis de maestría). Universidad Nacional, Heredia, Costa Rica.

Aranda, M. (2000). Huellas y otros rastros de los mamíferos grandes y medianos de México. Xalapa, México: Instituto de Ecología A. C.

Ávila-Nájera, D.; Rosas-Rosas, O.; Tarango-Arámbula, L.; Martínez-Montoya, J y Santoyo-Brito, E. (2011). Conocimiento, uso y valor cultural de seis presas del jaguar (Panthera onca) y su relación con éste, en San Nicolás de los Montes, San Luis Potosí, México. Rev. Mex. Biodiv., 82(3), 1020-1028. Recuperado de http:// www.scielo.org.mx/scielo.php?script=sci_arttext\&pid=S1870-34532011000300026

Beier, P. (1993). Determining the minimum habitat areas and habitat corridors for cougars. Conserv. Biol., 7, 94-108. DOI https://doi.org/10.1046/j.1523-1739.1993.07010094.x

Bustamante, A. (2008). Densidad y uso de hábitat por los felinos en la parte sureste del área de amortiguamiento del Parque Nacional Corcovado. (Tesis de maestría). Universidad Nacional, Heredia, Costa Rica.

Bustamante, A.; Moreno, R.; Rossel, E. y Artavia, A. (2014). Depredación del puma (Puma concolor) en un bosque Neotropical Centroamericano. Latin American Journal of Conservation, 4(1), 40-45. Recuperado de http://www.academia.edu/17176772/Depredaci\%C3\%B3n del_puma_Puma_concolor_en_un_bosque Neotropical_Centroam\%C3\%A9

Carrillo, E.; Wong, G. y Sáenz, J. (2002). Mamíferos de Costa Rica. Santo Domingo de Heredia. Costa Rica: INBio.

Dehustua, I. (2008). Descripción de los conflictos, percepciones, actitudes y usos tradicionales que poseen los pobladores rurales del departamento de Ayacucho, provincia de la mar, distrito de Anco sobre los mamíferos Alto Andinos (orden carnívora) durante el año 2006. (Tesis de grado). Universidad Nacional Agraria, Lima, Perú.

Echegaray, J. (2004). Percepción social de los grandes mamíferos en la cordillera Cantábrica (Montaña de Riaño, León). Galemys, 16(1),
25-40. Recuperado de http://www.alchata.es/ wpcontent/uploads/2008/10/apreciacion_social_grandes_carnivoros.pdf

Escamilla, A.; San Vicente, M.; Sosa, M. y Galindo-Leal, C. (2000). Habitat Mosaic, Wildlife Availability, and Hunting in the Tropical Forest of Calakmul, México. Conserv. Biol., 14, 1592-1601. DOI https://doi.org/10.1046/ j.1523-1739.2000.99069.x

Fa, J.; Aguilar, M.; Moya, A.; Duarte, J. y Yañez, J. (2013). Reflexiones sobre el impacto y manejo de la caza de mamíferos silvestres en los bosques tropicales. Ecosistemas, 22(2), 76-83. Recuperado de https://www.revistaecosistemas.net/index.php/ecosistemas/article/ view/740

Flores-Manzanero, A.; González-Pérez, G.; Vásquez-Dávila, M. y Manzanero-Medina, G. (2013). Conocimiento y usos de Odocoileus virginianus en Santo Domingo Tonalá, Oaxaca. Therya, 4:(1), 103-112. DOI https://doi. org/10.12933/therya-13-92

Galindo, R. (2012). Conocimiento tradicional y usos de los mamiferos terrestres silvestres en dos localidades indígenas del bosque tropical lluvioso del Norte de Oaxaca. (Tesis de grado). Universidad Nacional Autónoma de México.

Gallardo, G.; Núñez, A.; Pacheco, L. y Ruiz-García, M. (2009). Conservación del puma en el Parque Nacional Sajama (Bolivia): Estado poblacional y alternativas de manejo. Mastozool. Neotrop., 16(1), 59-68. Recuperado de http:// www.scielo.org.ar/scielo.php?script=sci_abstract\&pid=S0327-93832009000100006

García, D. (2011). Efectos biológicos de la fragmentación de hábitats: nuevas aproximaciones para resolver un viejo problema. Ecosistemas, 20(2-3), 1-10. Recuperado de https://www.revistaecosistemas.net/index.php/ecosistemas/ article/viewFile/18/12

Gil, R. y Guiascón, O. (2015). Uso de la Fauna Silvestre en la Comunidad Maya Villa De Guadalupe, Campeche, México. Etnobiología, 10(2), 1-11.

Lindenmayer, D.; Manning, A.; Smith, P.; Possingham, H.; Fischer, J.; Oliver, I., y McCarrthy, M. (2002). The Focal-Species Approach and Landscape Restoration: A Critique. Conserv. Biol., 16 (2), 338-345. DOI https://doi. org/10.1046/j.1523-1739.2002.00450.x

Nupp, T. y Swihart, R. (1996). Effect of forest patch area on population attributes of white-footed 
mice (Peromyscus leucopus) in fragmented landscapes. Can J. Zool.; 74, 467-472. DOI https://doi.org/10.1139/z96-054

Pacheco, C. (2003). Situación actual del venado cola blanca (Odocoileus virginianus) y caracterización socioeconómica de los vecinos del Parque Nacional Barra Honda, Costa Rica. (Tesis maestría). Universidad Nacional, Heredia, Costa Rica.

Parra-Colorado, J.; Botero-Botero, Á. y Saavedra-Rodríguez, C. (2014). Percepción y uso de Mamíferos silvestres por Comunidades Campesinas Andinas de Génova, Quindío, Colombia. Bol. Cient. Mus. Hist. Nat., 18(1), 78-93. Recuperado de http://www.scielo.org. $\mathrm{co} / \mathrm{pdf} / \mathrm{bccm} / \mathrm{v} 18 \mathrm{n} 1 / \mathrm{v} 18 \mathrm{n} 1 \mathrm{a} 05 . \mathrm{pdf}$

Racero-Casarrubia, A.; Vidal, C.; Ruiz, O. y Ballesteros, J. (2008). Percepción y patrones de uso de la fauna silvestre. Rev. Estud. Soc., (31), 118-131.

Rodas-Trejo, J.; Ocampo-Gonzáles, P. y Coutiño-Hernández, P. (2014). Uso de los mamíferos silvestres en el municipio de Copainalá, región Zoque, Chiapas; México. Quehacer Cientifico en Chiapas, 9(1), 3-9.

Reid, F. A. (1997). A field guide to the mammals of Central America and southeast Mexico. New York, USA: Oxford University Press.

Sandoval, I. y Castillo, M. (2011). Áreas protegidas de Costa Rica: Fragmentación, tamaño y forma. Métodos en Ecología y Sistemática, 6, 21-28. Recuperado de https://www.researchgate.net/profile/Maikol_Castillo_Chinchilla/ publication/305650741_Areas_protegidas de_Costa_Rica_Fragmentacion_tamano_y_ forma/links/5797841408aed51475e69c6f/ Areas-protegidas-de-Costa-Rica-Fragmentacion-tamano-y-forma.pdf
Salóm-Pérez, R.; Carrillo, E.; Sáenz, J. y Mora, J. (2007). Critical condition of the jaguar Panthera onca population in Corcovado National Park, Costa Rica. Oryx., 41, 51-56. DOI https://doi.org/10.1017/S0030605307001615

Sampieri, H.; Collado, C. y Lucio, P. (2010). Metodología de la investigación. Mc Graw Hill: Mexico D.F.

Schelhas, J. (2007). El valor de los fragmentos del bosque: enlazando el conocimiento social y ecológico. Corredores Biológicos: acercamiento conceptual y experiencias en América. San José, Costa Rica: Centro Científico Tropical-Universidad Nacional.

Stevens, V.; Polus, E.; Wesselingh, R.; Schtickzelle, N. y Baguette, M. (2004). Quantifying functional connectivity: experimental evidence for patch-specific resistance in the Natterjack toad (Bufocalamita). Landscape Ecology, 19, 829-842. DOI https://doi.org/10.1007/ s10980-004-0166-6

Tlapaya, L. y Gallina, S. (2010). Cacería de mamíferos medianos en cafetales del centro de Veracruz, México. Acta Zoológica Mexicana, 26(2), 259-277. DOI https://doi.org/10.21829/ azm.2010.262698

Troche, C. y Guarachi E. (2001). Análisis del cambio de cobertura y fragmentación del hábitat en el municipio de Independencia. Una propuesta metodológica simple para la identificación de áreas prioritarias de investigación biológi$c a$. Centro de Levantamientos Aeroespaciales y aplicaciones SIG para el Desarrollo Sostenible de los Recursos Naturales. Sinchi: Bogota.

Wainwright, M. (2005). The Mammals of Costa Rica. Cornell University Press. Comstock Publishing Associates: Ithaca, United States. 


\section{Anexo 1}

\section{No. de formulario:}

Encuesta sobre percepción de mamíferos medianos y grandes de los alrededores del Parque Nacional Barra Honda

Agradeceré mucho responda las siguientes preguntas sobre sus conocimientos de mamíferos presentes en los alrededores del Parque Nacional Barra Honda.

\section{Caracterización del entrevistado}

Información general

Género:

Nivel de estudios:
a) Sin estudios
b) Primaria
c) Secundaria (colegio)
d) Superiores

Fecha: Profesión u oficio:
$/ 2011$

\section{Distrito:}

Barrio o caserío:

Distancia aproximada a PNBH:

¿Desde hace cuánto tiempo reside/labora en la zona?

\section{Conocimiento popular}

¿Ha observado usted últimamente alguno de estos animales?

\begin{tabular}{|l|l|}
\hline Zorra gris & \\
\hline Coyotes & \\
\hline Pizote & \\
\hline Perica lijera, perezoso & \\
\hline Zorro hediondo & \\
\hline Caucel, tigrillo & \\
\hline Tolomuco, viejo del monte & \\
\hline Zorricí & \\
\hline Mapache & \\
\hline Guatuza, cherenga & \\
\hline Mono carablanca & \\
\hline Zorro de balsa & \\
\hline
\end{tabular}

\begin{tabular}{|l|l|}
\hline Tepezcuintle & \\
\hline Saíno & \\
\hline Ardilla, chiza & \\
\hline Danta, tapir & \\
\hline Cabro de monte & \\
\hline Puma león & \\
\hline Venado cola blanca & \\
\hline Jaguar, tigre, pantera & \\
\hline Oso hormiguero & \\
\hline Cusuco, armadillo & \\
\hline Mono congo & \\
\hline Manigordo, ocelote & \\
\hline
\end{tabular}

\section{Percepciones}

¿Existe algún mamífero que sea abundante en la zona?
a) Sí
b) No
c) No sabe
d) No contesta

¿Cuál(es)? 
¿En la actualidad existe(n) algún(os) animal(es) que sea(n) escaso(s)?
a) Sí
b) No
c) No sabe
d) No contesta

¿Cuál(es)?

¿En su comunidad se mantienen mamíferos en cautiverio?
a) Sí
b) No
c) No sabe
d) No contesta

¿Por qué los utilizan?

Le parecen animales bonitos o atractivos

Es un regalo que le hicieron

Por costumbre

Por compañía

\section{Problemática con mamíferos}

¿Algún mamífero le ha causado daños a usted o a sus bienes?
a) Sí
b) No
c) No sabe
d) No contesta

¿A qué le han causado daños?
a) Cultivos
b) Ganado
c) Propiedadese) Otras personas

¿Sabe usted si se ha presentado algún ataque de algún mamífero a personas de la comunidad?
a) Sí
b) No
c) No sabe
d) No contesta

¿Cuál(es) mamífero(s)?

\section{Motivaciones de la caza}

¿Conoce si en la zona se realizan actividades de cacería?
a) Sí
b) No
c) ¿Por qué las realizan?

i. Subsistencia

ii. Actividad familiar

iii. Deporte

iv. Curiosidad

v. Comercio

¿Realiza usted dichas actividades en su comunidad o en los alrededores?
a) Sí
b) No
c) No sabe
d) No contesta

¿Cuánto tiempo le toma llegar hasta un lugar donde se puedan capturar mamíferos?
a) De 1 a 2 horas
b) De 3 a 5 horas
c) De 6 a 10 horas
d) Más de 12 horas

¿Sabe usted si la cacería de mamíferos es una actividad común en su comunidad?
a) Sí
b) No
c) No sabe
d) No contesta

¿Cuántas especies de mamíferos se pueden capturar en esta zona?
a) De 1 a 2 especies
b) De 3 a 5 especies
c) De 6 a 10 especies
d) Más de 10 especies 


\section{Protección}

¿Conoce usted si algún mamífero presente en la zona está protegido por ley?
a) Sí
b) $\mathrm{No}$
c) No sabe
d) No contesta

¿Se deben proteger?
a) Sí
b) No
c) No sabe
d) No contesta

¿A quién le corresponde la protección de los mamíferos de la zona?
MINAET
b) Comunidad
c) Gobierno
d) Organizaciones privadas

¿Considera usted que es importante la protección que se le pueda brindar a los mamíferos de la zona?
a) Sí
b) No
c) No sabe
d) No contesta

Conocimiento popular de los mamíferos del Parque Nacional Barra Honda, Nicoya, Costa Rica (Maikol CastilloChinchilla y otros) por Revista Uniciencia se encuentra bajo una Licencia CreativeCommons Atribución-NoComercialSinDerivadas 3.0 Unported. 